\title{
Reflectivity Optimization of the SPR Graphene Sensor
}

\author{
S. Benaziez ${ }^{1, *}, Z^{\prime}$. Dibi ${ }^{2, \#}$ and N. Benaziez ${ }^{1}$ \\ ${ }^{1}$ Physics Department, Faculty of Material Sciences, University of Batna1, 05000, Algeria \\ ${ }^{2}$ Electronic Department, Faculty of Science of Engineering, University of Batna2, 05000, Algeria
}

Received: 10 October 2017; accepted: 14 February 2018

\begin{abstract}
In this study, the optimization of the surface plasmon resonance (SPR) sensor based on graphene-silver substrate was investigated. We simulated the reflection spectrum that depends on the metal thickness and the number of graphene layers. The addition of a certain number of graphene layers based on the knowledge that silver oxidation decreases the sensitivity of the sensor improved the sensitivity $\mathrm{S}_{\mathrm{RI}}$, while the detection accuracy decreased. To optimize the sensor performance, the investigation focused on monolayer graphene. Furthermore, genetic algorithms were used to optimize the SPR biosensor reflection by adjusting the coefficients of the system, which are the incidence angle and metal layer thickness.
\end{abstract}

Keywords: surface plasmon resonance (SPR), biosensor, graphene, genetic algorithm

\section{Introduction}

Many recent works have undertaken the manufacture and development of biosensors using various sensing methods. Surface plasmon resonance (SPR) is one among them. This methode investigates a wide range of different putative interactions with a variety of biomolecules in real time without labelling [1,2].

Surface plasmon is a charge-density oscillation propagating along the interface between metal and dielectric medium. The evanescent wave of the surface plasmon is affected and determined by the properties of the metal and dielectric, and it can be excited by a light. The Kretschmann configuration is one of the techniques that are used in this excitation [3]. It is based on the observation of the reflected light spectrum obtained either by "angular interrogation" or "wavelength interrogation". Generally, in SPR sensors the angular approach is the preferred due to its low angular resolution [4-6]. It is based on the measurement of changes in the resonant angle extracted from the curve when changing the angle of incidence and keeping the wavelength constant. In addition to the resonance angle, this spectrum is characterized by two other factors are half width at half maximum (FWHM) and reflection minimum $R_{\min }[5]$.

The noble metals $[7,8]$ as $\mathrm{Ag}$ and Au are more commonly used to support the propagation of surface plasmon polariton waves (SPP) at visible light wavelengths $[4,9,10]$. How-

This is an open-access article distributed under the terms of the Creative Commons Attribution-NonCommercial 4.0 International License (https://creativecommons.org/licenses/by-nc/4.0/), which permits unrestricted use, distribution, and reproduction in any medium for non-commercial purposes, provided the original author and source are credited, a link to the CC License is provided, and changes - if any - are indicated.

Corresponding authors, ${ }^{*}$ E-mail: safia.mag1@gmail.com, ${ }^{\#}$ E-mail: zohirdibi@yahoo.fr 
ever, silver substrates seems to be the most appealing, because it provides in the metal-sensing layer interface a high value of electric field enhancement with low imaginary part of refractive index [11]. When compared with gold, the plasmon coupling in the silver has a sharper angular resonance peak [12], but it presents a drawback that it can be easily oxidized and the poor chemical stability thus affecting the performance of SPR-sensors [11, 12]. Many of methods were designed in an attempt to overcome this problem, for example the use of bimetallic silver-gold layers [13, 14], a thin oxide films $\mathrm{TiO}_{2}$ or $\mathrm{SnO}_{2}$ [15-17], In addition to thin films of carbon such as the amorphous carbon [18], amorphous carbonated silicon $\left(\mathrm{a}-\mathrm{Si}_{0.63} \mathrm{C}_{0.37}\right)$ [19-21] and the newer one is, graphene [13].

Graphene is a carbon nanomaterial with two-dimensional (2D) sheet structure composed of $\mathrm{sp}^{2}$ carbon atoms arranged in a honeycomb lattice [21]. It has been proposed as an addition of layers that can prevent the oxidation of silver metal and at the same time have a high capacity of efficient adsorption of biomolecules $[11,22]$, due to their high surface area and rich $\pi$ conjugation structure. This $\pi$ orbitals form a dense cloud that blocks the gap within its atomic rings. Thus preventing the oxygen from interacting with them [13, 21-23]. Further, the graphene layers possesses very fascinating properties include the optical conductivity in the visible-infrared range, the lowest resistivity, high electron mobility, and relatively low cost $[1,24,25]$.

Based on these properties, Wu et al. [26] have investigated graphene-on-gold SPR biosensor and demonstrated that the biosensor with $L$ graphene layers is more sensitive than the conventional gold thin film SPR biosensor. The improved sensitivity is due to the increased adsorption of biomolecules on graphene. Maharana et al. [27] incorporated graphene as dielectric over layer on SPR active metal Ag for enhancing electric field at the sensing layer interface. The proposed sensor shows enhanced performance as compared to widely reported $\mathrm{Au}$ on Ag configuration. Choi et al. [28] have demonstrated that sensitivity of graphene-onsilver SPR biosensor can be greater than the sensitivity of the conventional gold-film-based biosensor. as well as the graphene layers prevent silver oxidation. Islam et al. [4] have studied the enhancement of the sensitivity and adsorption efficiency of a localized surface plasmon resonance (LSPR) biosensor that includes a layer of graphene sheet on top of the gold layer. The LSPR graphene biosensor has better sensitivity with lower operating wavelength and larger number of graphene layers have also been studied. In other works [29], Djeffal et al. proposed a new graphene-based sensor called Dielectric Modulated Graphene Field Effect Transistor (DMG-FET), for high-performance biomolecule sensing applications.

In this paper, we shall present an ultra-stable high performance SPR sensor based on a graphene on Ag configuration, which depends on the thickness of the silver layer and the number of graphene layers.

In the light of new optimization techniques, we used a genetic algorithm (GA), which is a heuristic method. It was found to be a powerful tool to solve and optimize problems based on the survival of the fittest. The theory of the evolution of species in their natural environment is an artificial transposition of basic concepts of genetics and survival of laws set forth by Charles Darwin: the most adapted individuals survive and reproduce. These same mechanisms will be used in the implementation of the GA [30,31], which will be validated by a problem which searches for the optimum reflectivity of graphene SPR biosensor. The optimization procedure starts with the acquisition of different data related to the parameters in the problem, which are mainly the interval of incidence angle and the metal thickness layer. A good agreement is observed between the theoretical generalized multi-layer model and the optimization result. The obtained results allow us to conclude that the reflection fitness function is minimal whatever the number of iterations. 
The SPR sensor with the Kretschmann configuration consists of a glass prism, a silver film which can experimentally deposited onto the prism by hot evaporation with depositing rate of $0.5 \mathrm{~A}^{\circ} / \mathrm{s}$ at a vacuum of $1 \cdot 10^{-6}$ Torr [32], and a sample in sequence [33]. Kretschmann configuration is a simple model for excitation SPR, that understood with observed the SPR curve (the change reflected intensity $R$ versus function angle incidence). When the light wave traverses the prism it generates an evanescent wave that propagates and penetrates in the metal surface with propagation constant $k_{x}$ as in Eq. (1).

$$
k_{x}=\left(2 \pi / \lambda_{0}\right) n_{1} \sin \theta
$$

where $\theta$ is the angle of incidence of light with the normal to the interface, and $n_{1}$ is the prism refractive index.

For a specific angle of incidence, $k_{x}$ can be adjusted to match the SPP and its interaction with the light wave influences the reflectivity $R$ [26]. In the simulation of the SPR biosensor with a graphene-on-silver substrate (shown in Fig. 1a), we used the same previous model to which a new element was added: the graphene layer is sandwiched between the silver and a sensing film layer, to absorb the biomolecules (e.g. ssDNA ) present in the water. Practically, The transfer of graphene onto silver SPR interfaces can be achieved in several ways as dry transfer technique. The transfer of large-area graphene grown by chemical vapour deposition (CVD) onto metal substrates is an attractive technique that enables us the control of transferred graphene layers number [13].

The composition of the sensor is as follows:

- A tunable laser $(632.8 \mathrm{~nm})$,

- A prism made of silica glass $B K 7\left(n_{1}=1.515\right.$, depth $\left.d=\infty\right)$,

- A silver metal layer whose dielectric function follows the Lorentz-Drude model.

The relationship is given by

$$
\varepsilon_{m}(\omega)=1-\left(\frac{f_{0} \omega_{p}^{2}}{\omega\left(\omega+\mathrm{i} \Gamma_{0}\right)}\right)+\sum_{j=1}^{k} \frac{f_{j} \omega_{p}^{2}}{\omega_{j}^{2}-\omega^{2}+\mathrm{i} \omega_{j}},
$$

where $\omega=(2 \pi c / \lambda)$ is the light frequency, $\omega_{p}$ the plasma frequency, $k$ the number of oscillators with frequency $\omega_{j}$, strength $f_{j}$, and lifetime $\left(\Gamma_{j}\right)^{-1}$; while $f_{0} \omega_{p}{ }^{2}$ is the plasma frequency associated with intra-band transitions with oscillator strength $f_{0}$ and damping constant $\Gamma_{0}$ [33].

- A graphene layer of refractive index $n_{g r}=2.7+\mathrm{i} 1.4$ [27] and thickness $d=L \times 0.34 \mathrm{~nm}$

[28], where $L$ is the number of graphene layers.

- A bio-sensing layer $n_{s}=1.33$.

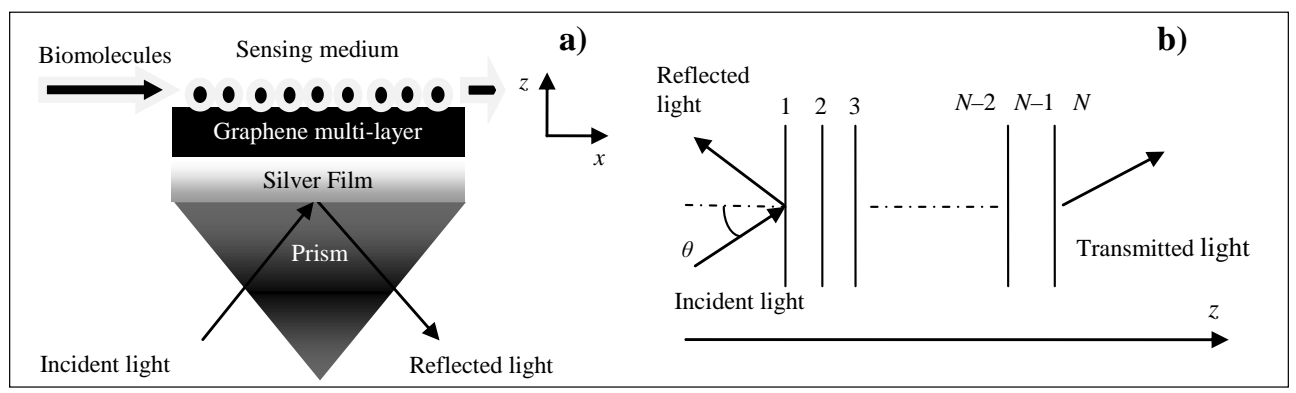

Fig. 1. (a) Configuration of the graphene-on-silver SPR biosensor [1]. (b) Schematic diagram of the multi-layer model [33] 
We will use a generalized $N$-layer model $[1,33,34]$, shown in Fig. $1 b$. To generate a SPR curve and obtain the reflectivity of the incident p-polarized light [27], all the layers are stacked along the $z$ direction. The arbitrary medium layer is defined by the thickness $\left(d_{k}\right)$, dielectric constant $\left(\varepsilon_{k}\right)$, permeability $\left(\mu_{k}\right)$, and refractive index $\left(n_{k}\right)$. The tangential fields at the first $z=z_{1}=0$ and at the last boundary $z=z_{N-1}[1]$ are related by

$$
\left[\begin{array}{l}
U_{1} \\
V_{1}
\end{array}\right]=M\left[\begin{array}{c}
U_{N-1} \\
V_{N-1}
\end{array}\right]
$$

$U_{1}, U_{N-1}$ are the components of electric field at the boundary of the first and $N$ th layers of structure, respectively, while $V_{1}, V_{N-1}$ are the components of magnetic field and $M$ is the characteristic matrix of this structure, which is given by

$$
M=\prod_{K=2}^{N-1} M_{K}
$$

with

where

$$
M_{k}=\left[\begin{array}{cc}
\cos \beta_{k} & -\mathrm{i} \sin \beta_{k} / q_{k} \\
-\mathrm{i} q_{k} \sin \beta_{k} & \cos \beta_{k}
\end{array}\right] \text {, }
$$

and

$$
q_{k}=\left[\varepsilon_{k}+n_{1}^{2} \sin ^{2} \theta\right]^{1 / 2} \varepsilon_{k}^{-1}
$$

$$
\beta_{k}=\left(2 \pi d_{k} / \lambda\right)\left[\varepsilon_{k}+n_{1}^{2} \sin ^{2} \theta\right]^{1 / 2} \text {. }
$$

The amplitude reflection coefficient $\left(r_{p}\right)$ for p-polarized light is:

$$
r_{p}=\frac{\left(M_{11}+M_{12} q_{N}\right) q_{1}-\left(M_{21}+M_{22} q_{N}\right) q_{N}}{\left(M_{11}+M_{12} q_{N}\right) q_{1}+\left(M_{21}+M_{22} q_{N}\right) q_{N}} .
$$

The reflection coefficient of the $N$-layer model for p-polarized light is:

$$
R_{p}=\left|r_{p}\right|^{2}
$$

For an enhanced performance of biosensors, the sensitivity is an important issue for its application. It depends on a number of factors such as the operating wavelength and material characteristics, including the refractive index of dielectric layer, refractive index of prism, metal film, and film thickness, etc. They are selected to optimize the resonance condition [2]. The sensitivity of the sensor is defined as [1]:

$$
\mathrm{S}=\left(\Delta \theta_{\text {res }} / \Delta n\right)
$$

where the change in the resonance angle is $\Delta \theta_{\text {res }}$, corresponding to a change $\Delta n$ in the refractive index of sensing layer. The resonance angle $\left(\theta_{\text {res }}\right)$ corresponding to the minimum reflection coefficient $\left(R_{\min }\right)$ is determined from the $\mathrm{R}-\theta$ curve.

Another important requirement for a high-performance SPR sensor is its high detection accuracy (which is inversely proportional to the FWHM). It can be noted that the precision of the measured SPR angle depends strongly on the width of the SPR curve $[11,35]$. 


\section{Design parameters and results}

\subsection{Effect of film thickness on the SPR-sensor}

Without graphene layers: The metal thickness is a very important parameter in surface plasmon resonance. It defines the maximum dynamic and shape of the plasmon [7, 33].

Figure 2 shows the calculated results as a series of SPR curves for silver thickness increasing in steps of $10 \mathrm{~nm}$ changing from $10 \mathrm{~nm}$ to $100 \mathrm{~nm}$. The resonance angle $\left(\theta_{\text {res }}\right)$ increases in the range of 53-67. $8^{\circ}$.

The thickness of the silver layer is optimized to such an extent that the resonance dip in the SPR curve becomes the sharpest with a reflection minimum close to zero. So the best value is $51.26 \mathrm{~nm}$, which can be precisely extracted from the curve of reflectivity as a function of the silver layer thickness (inset of Fig. 2).

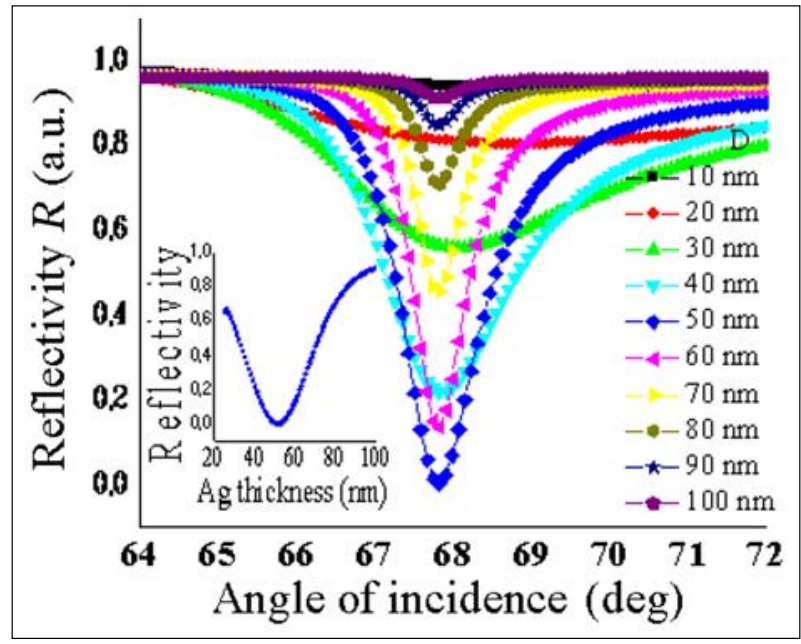

Fig. 2. Silver thickness layer effect on the reflection spectrum

It is not necessary a very high thickness, because as shown in Fig. 2, the maximum is around $100 \mathrm{~nm}$. Above this thickness, the evanescent wave due to the total reflection on the prism cannot cross the metal to generate plasmon at the metal/dielectric interface. Thus, the minimum of reflectance moves away from zero and the SPR reflection spectra start to broaden. In the case of a thin metal $(10-30 \mathrm{~nm})$, it tends to limit the total reflection on the prism because there is not enough metal to absorb the incident wave and oscillate plasmons. For these small thicknesses, it is seen that the reflectivity on one hand does not drop to a minimum of zero. On the other hand, the resonance is large, which reduces the sensor sensitivity. Furthermore, it was found that the variation in metal thickness decreases the amplitude of the resonance peak, which means a reduction in the transfer of incident energy to surface plasmons. The optimization of the thickness of the metal is important to work in the best coupling conditions and have the best sensitivity of the SPR sensor [7, 33].

With graphene layers: In order to study the effect of the film thickness on the SPR graphene sensor, we should take into account the number of graphene layers. Figure $3 a$ illustrates that when the number of graphene layers $L$ increases, it leads to a shift of the resonance peak, a 


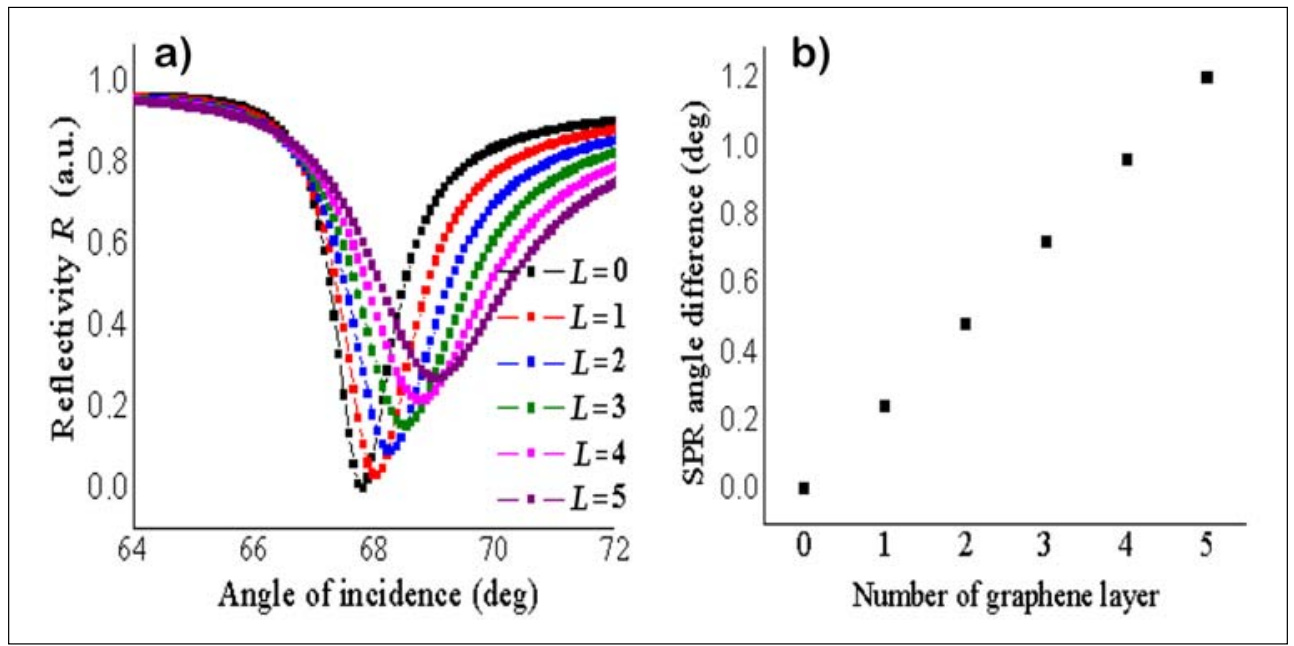

Fig. 3. (a) SPR reflectance and (b) change in the SPR angle as the number of graphene layers increases (0-5 layers), with sensing layer refractive index of 1.330

variation of its amplitude, and a change and modification of the width of its peaks which become wider respectively. For five graphene layers, the plasmonic angles shift from $67.81^{\circ}$ to $69.02^{\circ}$. Therefore, it was shown in Fig. $3 \mathrm{~b}$ that the change of the position of the minimum surface plasmon varies linearly with the number of additional graphene layers.

Additionally, one can note that the reduction in the amplitude of the resonance dip is a direct consequence of the change in the propagation constant of surface plasmons as a function of the number of graphene layers. The latter becomes different of the wave vector tangential component of incident light and hence the resonance condition is not fulfilled (i.e., SPs become damped) [10,13]. To minimize this effect, we optimize the silver layer thickness that decreases when the number of graphene layers increases (see Table 1).

The silver thickness for which the reflectivity is zero represents the optimum thickness. Figure $4 a$ illustrates the variation of reflectivity as a function of silver layer thickness for various numbers of graphene layers.

Table 1. Optimized values of silver thickness in terms of the number of graphene layers for corresponding changes in the resonance angle for zero reflectivity in the SPR. The refractive index of the sensing film was 1.33

\begin{tabular}{cccc}
\hline $\begin{array}{c}L \\
\text { graphene } \\
\text { layers number }\end{array}$ & $\begin{array}{c}D_{\mathrm{Th}} \\
\text { silver thickness } \\
\left(10^{-9} \mathrm{~nm}\right) \text { theory }\end{array}$ & $\begin{array}{c}\theta_{\mathrm{Th}} \\
\text { theoretical resonance } \\
\text { angle (degree) }\end{array}$ & $\begin{array}{c}R_{\min (\mathrm{Th})} \\
\text { minimum reflection } \\
\text { coefficient (a.u.) }\end{array}$ \\
\hline 0 & 51.26 & 67.817 & $6.4485 \mathrm{E}-004$ \\
1 & 47.22 & 68.058 & $6.3873 \mathrm{E}-004$ \\
2 & 44.23 & 68.298 & $4.2867 \mathrm{E}-004$ \\
3 & 41.84 & 68.538 & $2.0990 \mathrm{E}-004$ \\
4 & 39.85 & 68.778 & $5.8913 \mathrm{E}-005$ \\
5 & 38.14 & 69.099 & $5.5688 \mathrm{E}-007$ \\
\hline
\end{tabular}




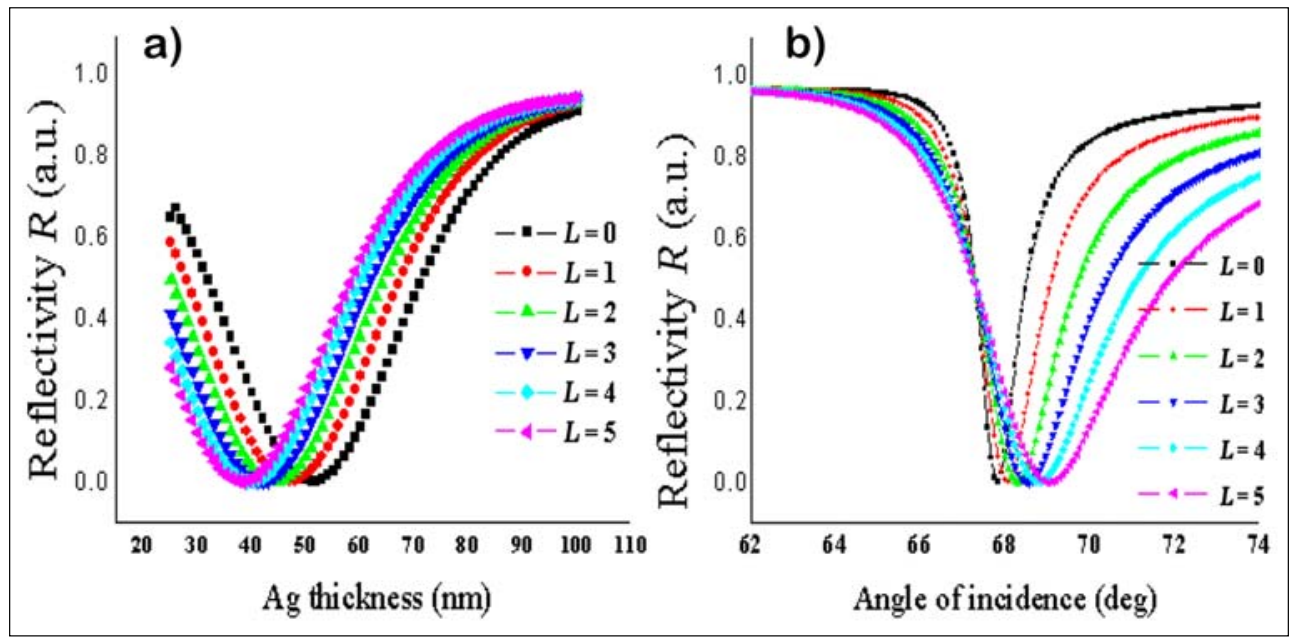

Fig. 4. (a) Variation of reflectivity with respect to the thickness of the silver layer. (b) Angular reflectivity spectra corresponding to optimum values of the thickness of silver for graphene layers (1-5 layers)

Figure $4 b$ shows the angular spectra of reflectivity corresponding to these optimum values. It is noted that the optimization of the silver thickness provides a shallower dip in the reflectivity curve. The results are summarized in Table 1.

\subsection{Graphene layers effect on detection accuracy and SPR-sensor sensitivity enhancement}

It is important to make a good choice for the maximum number of graphene layers, which must be fixed for the high performance of the biosensor. This fixed number must be taken in order to give a high sensitivity enhancement and a high detection accuracy.

In Fig. $5 a$, the sensitivity enhancement as a function of the number of graphene layers $L$ after the absorption of biomolecules is plotted, assuming the same refractive index change $\Delta n=0.005$.It is found that adding more graphene layers allows an increase in sensitivity. The

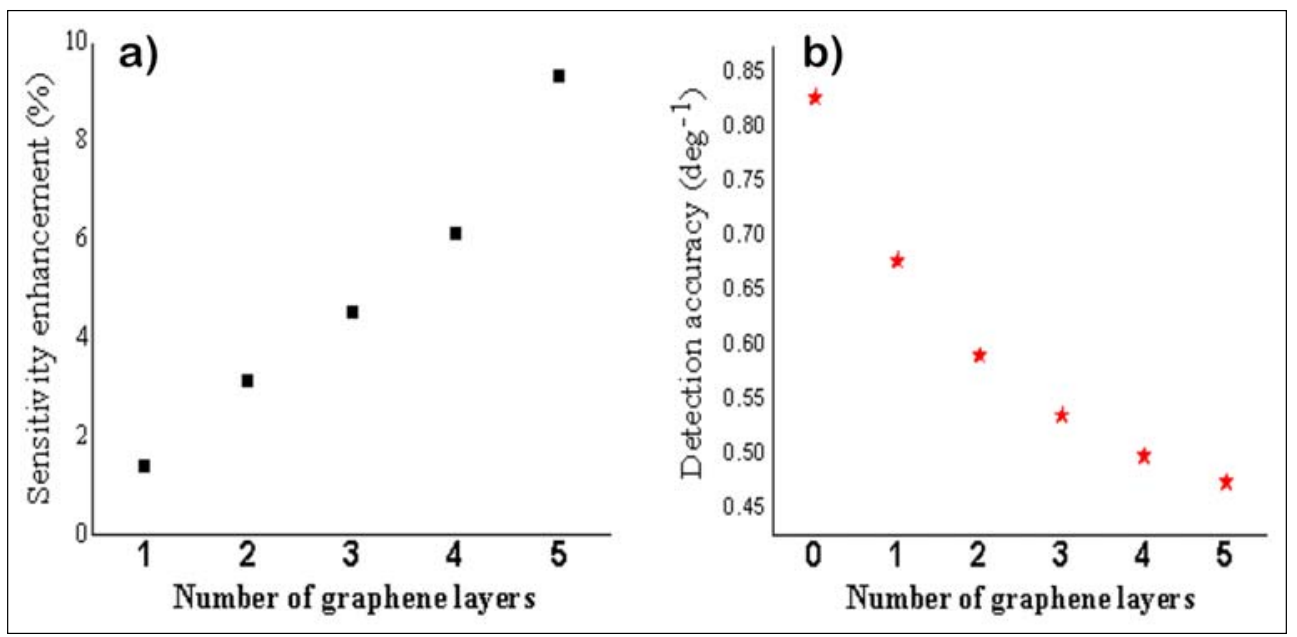

Fig. 5. (a) Variation in sensibility enhancement and (b) detection accuracy versusthe number of graphene layers ( $1-5$ layers) 


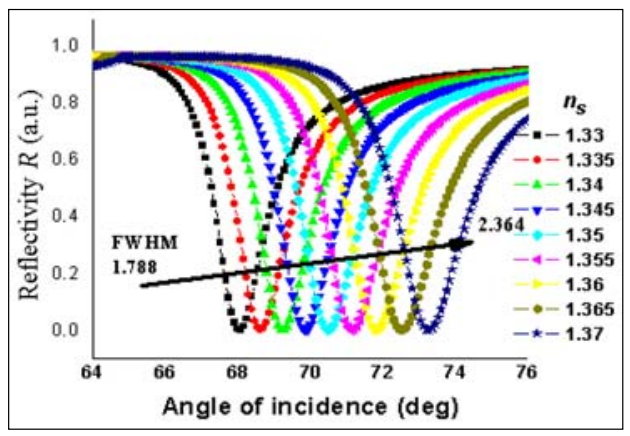

Fig. 6. SPR reflection spectra for monolayer graphene layers as a function of the incident angle with various values for sensing medium refractive index

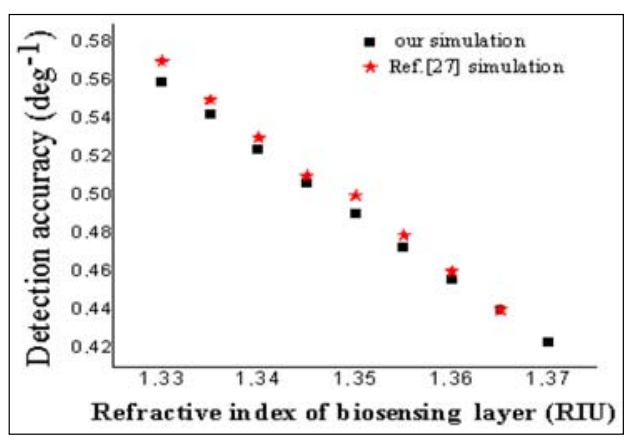

Fig. 7. Variation of detection accuracy with sensing layer refractive index for monolayer graphene at two wavelengths $(632.8 \mathrm{~nm}$ in our study and $633 \mathrm{~nm}$ in Ref. [27]) for comparison

sensitivity enhancement value can be $1.4 \%$ for the monolayer graphene biosensor, $3.1 \%$ $(L=2), 4.5 \%(L=3), 6.1 \%(L=4)$, and 9.3\% $(L=5)$. Graphene is a good absorbent of biomolecules, since the increase of graphene layers means the increase in the absorption of biomolecules, thus an increase in sensitivity [10]. Figure $5 b$ shows the effect of the number of graphene layers on detection accuracy. We can see that the increase in the number (or thickness) of graphene layers causes a reduction in detection accuracy from 0.818 to 0.221 degree $^{-1}$.The reason of this decrease is the breadth in the curve, as a result of the damped extension of SP field into the graphene layer, due to the amortization in the absorption of surface plasmon at the silver/graphene interface, which is also due to the optical properties of graphene (non-zero imaginary refractive index part) $[10,13,26]$. Finally, it is clear that the use of graphene as a protective layer in the Kretschmann configuration based on silver becomes important if the number of layers decreases. The following work will address the case of a monolayer graphene layers on a thin silver film.

For the monolayer graphene SPR sensor, we have theoretically demonstrated that the refractive index of the sensing film has an important influence on the reflection spectra and detection accuracy, because any change in the properties of the dielectric medium are determined by the surface plasmon evanescent waves [5]. Figure 6 shows that when the sample refractive index $n_{\mathrm{s}}$ changes by $0.04 \mathrm{RIU}$ (from 1.33 to 1.37), the width of resonance values increase from 1.7881 to 2.3642 . This increase in the width of the curve leads to difficulties in a $\theta_{\mathrm{SPR}}$ measurement $[10,26]$, and therefore to a decrease in the accuracy detection of the refractive index measurement, as depicted in Fig. 7. The results are compared with those obtained by Maharana et al. (2013) [27] for an operating wavelength of $633 \mathrm{~nm}$. As it can be seen, they are in good agreement with the same change of sensing layer refractive index.

\section{Genetic optimization}

A developed analytical expressions can be exploited to formulate the objective functions to optimize hence the device performance using the most known metaheuristic techniques known as genetic algorithm approach [36]. The GAs are searching processes based on the laws of natural selection and genetics, from Darwinian's theory of survival of the fittest. They use probabilistic transition rules instead of deterministic rules. The GAs are iterative stochastic algorithms that work upon groups of codified points over an initial population. The population of potential solutions is represented by individuals or chromosomes (parents) that 
evolve through successive iterations called generations. The fittest individual will survive a generation and generate offspring, which is evaluated by the quality of its fitness function and with a similar fashion to their parents. This is the last individual, which might be stronger. In order to allow a population of solutions to converge to optimal solutions, we follow iteration process steps based on the genetic triangle: reproduction, evaluation, and selection [37-40]. In this work, we will be searching for the best value of resonance angle and thickness film, which will give the optimum reflection fitness function. The various implementation shell steps of this methodology are summarized below.

Initialization: The first phase of the AG consists to choose randomly the initial population that contains a group of individuals whom are the different operators of an AG that would be applied.

Selection: Selection is supposed to be able to compare each chromosome in the population. Good chromosomes and forms a mating pool it is likely to be selected to reproduce, the most useful method is the roulette wheel scheme (RWS).

Crossover: Crossover operator is applied to the mating pool with the hope that it creates a better offspring and that is through exchanging information among strings of the mating pool with a crossover probability $P_{c}$. A string point crossover operator is used here which is performed randomly by choosing a crossing site along the string and by exchanging all bits on either side of the chosen site.

Mutation: This operator randomly flips some of the bits in a chromosome, i.e. 1 is mutated into 0 and vice versa. Mutation is also used to maintain diversity in the population.

Fitness evaluation: The evaluation phase permits to determine whether the optimum is obtained or re-executed another cycle of the AG. Each individual is codified then evaluated using the objective functions [30, 37-40].

In this work, we focus on optimizing the physical and geometrical design parameters of our proposed device (i.e. the value of resonance angle and thickness film) with the aim of reaching their greatest performance (i.e. the value of the reflection fitness function). The GAbased approach can be appropriate for investigating and optimizing the proposed SPR graphene sensor design through optimized the minimizing the reflection $R$. Typically, based on matrix for an $N$-layer system, the objective function can be expressed by the following equation:

$$
\text { Fitness }(X)=R \text {, }
$$

where $X$ denotes the design physical and geometrical vector presented by: $X=(\theta, D)$. Evidently, a global optimization problem based on mono-objective procedure is mainly illustrated by a candidate solution in well defined search space and a set of constraints that should be respected [36]. These constraints can be given in our case by the following conditions:

$-x \in\left[x_{i, \min }, x_{i, \max }\right], x_{i} \in X$ (each design variable should be confined within a given range). In this framework, the intervals of incidence angle $\theta_{\text {inc }}$ is $[10 \ldots 90]$ (degree) and thickness metal layer $D$ is $[30 \ldots 100]$ (nm), respectively,

$-L$ is the number of graphene layers and is equal to one, 


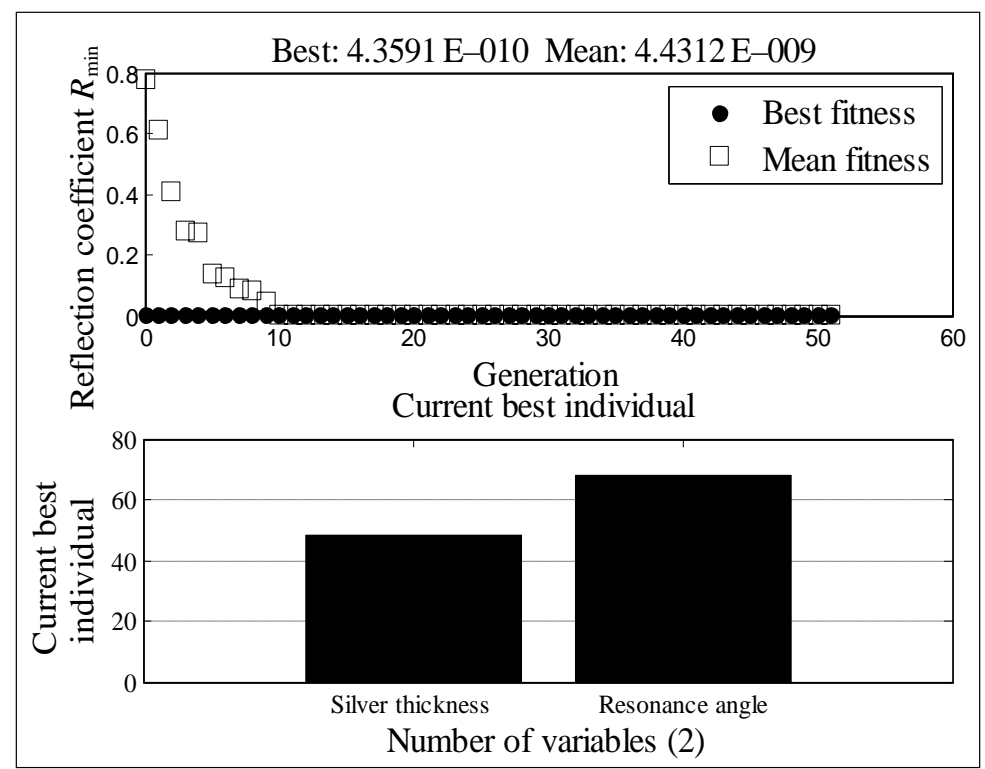

Fig. 8. Best fitness plot for the minimization of the reflection function. The plots show the results from Table 2

Table 2. Summary of results of the GA application

\begin{tabular}{ll}
\hline Population size & 20 \\
Number of generations & 51 \\
Method used in selection & Roulette wheel scheme (RWS) \\
Crossover probability $P_{c}$ & 0.8 \\
Mutation probability $p_{m}$ & 0.01 \\
Optimum minimum reflection coefficient $R_{\text {min }}$ (a.u.) & $4.3591 \mathrm{E}-10$ \\
Resonance angle optimize $d \theta_{\mathrm{Ag}}(\mathrm{SPR})($ degree $)$ & 67.927 \\
Silver thickness optimized $D_{\mathrm{Ag}}\left(\cdot 10^{-9} \mathrm{~nm}\right)$ & 48.404 \\
Execution time $(\mathrm{s})$ & $\approx 0.732634$ \\
\hline
\end{tabular}

for which a quick stabilization of the objective function can be achieved where the estimated error is extremely small. After performing the optimization procedure, we can notice the outstanding capability of the proposed GA-based optimization for reaching the global optimum which is depicted in Fig. 8. In this context, this figure shows the evolution of the fitness function, Fitness $(X)$, against the generation number, where it is obvious that a quick stabilization of the objective function nearly after 51 iterations is achieved.

The convergence of the GA for optimum reflectivity is represented by the best parameters found in Table 2. The choice of a population with a small size leads to a fast resolution, even with a large number of evaluations of the fitness function, and it is effective in terms of time. Therefore, no change in the metal thickness or resonance angle after these values of size was noticeable. The obtained results considering these parameters are summarized in Table 2. 


\section{Conclusion}

We have presented in this paper, using numerical simulations, the influence of two parameters on the material characteristics of the SPR-sensor based on graphene and silver substrates. These parameters are the thickness of silver layer and the number of graphene layers on the shape of reflectivity curve. For the conventional SPR biosensor, it is found that a deviation of the metal thickness compared with the optimum value $D_{\text {opt }}=51.26 \mathrm{~nm}$ causes a shift in the resonance peak, a change of its width, and a variation of its amplitude. After the addition of graphene layers, which are used as a layer to protect the silver metal from oxidation, the reflectivity does not drop to a minimum of zero because the surface plasmons become damped. This leads to the optimization of the silver layer thickness, which decreases when the number of graphene layers increases to enable the realization of the resonant condition and thereby increase the efficiency of surface plasmons excitation. On the other hand, we have presented a discussion concerning the enhanced performance of SPR graphene sensor, which is assessed in terms of sensitivity and detection accuracy. We have found that the sensitivity enhancement value can be increased from $1.4 \%$ for the monolayer graphene to $9.3 \%$ for five layers, but the detection accuracy decreased from 0.78 to 0.46 degree $^{-1}$. Afterwards, we focused on the case of a monolayer graphene on a thin silver film used in the implementation of the GA. Its application has allowed us to obtain the searched results related to the problem of determination of the optimum reflected by optimal reflection, as well as seeking the best parameters allowing us to determine them according to the incidence angle and metal thickness.

\section{References}

[1] Verma R., Gupta B. D., Jha R. (2011) Sensitivity enhancement of a surface plasmon resonance based biomolecules sensor using graphene and silicon layers. Sens. Actuators B160 623-631.

[2] Gupta G., Kondoh J. (2007) Tuning and sensitivity enhancement of surface plasmon resonance sensor. Sens. Actuators B122 381-388.

[3] Akimoto T., Sasaki S., Ikebukuro K., Karube I. (2000) Effect of incident angle of light on sensitivity and detection limit for layers of antibody with surface plasmon resonance spectroscopy. Biosens. Bioelectron. 15(7) 355-362.

[4] Islam M. S., Kouzani A. Z., Dai X. J., Michalski W. P., Gholamhosseini H. (2011) Comparison of performance parameters for conventional and localized surface plasmon resonance graphene biosensors. International Conference of the IEEE EMBS Boston, Massachusetts USA (IEEE, 2011), pp. 1851-1854.

[5] Özdemir S. K., Turhan-SayanG. (2003) Temperature effects on surface plasmon resonance: design considerations for an optical temperature sensor. J. Lightwave Technol. 21(3) 805-814.

[6] Wohlhueter R. M., Parekh K., Udhayakumar V., Fang S., Lal A. A. (1994) Analysis of binding of monoclonal antibody to a malarial peptide by surface plasmon resonance biosensor and integrated rate equation. J. Immunol. 153(1) 181-189.

[7] Lecaruyer, P., Canva, M., and Rolland, J. (2007) Metallic film optimization in a surface plasmon resonance biosensor by the extended Rouard method. Appl.Opt. 46(12) 2361-2369.

[8] Islam M. S., Kouzani A. Z., Dai X. J., Michalski W. P. (2010) Investigation of the effects of design parameters on sensitivity of surface plasmon resonance biosensors. Biomed. Signal Process. Control 6(2) 147-156.

[9] Green R. J., Frazier R. A., Shakeshe K. M., Davies M. C., Roberts C. J., Tendler S. J. B. (2000) Surface plasmon resonance analysis of dynamic biological interactions with biomaterials. Biomaterials 21(18) 1823-1835.

[10] Maharana P. K., Jha R. (2012) Chalcogenide prism and graphene multilayer-based surface plasmon resonance affinity biosensor for high performance. Sens.Actuators B169 161-166.

[11] Bharadwaj S., Maharana P. K., Das R., Jha R. (2012) Effect of chalcogenide glass and plasmonic metal on electric field enhancement in surface plasmon resonance sensor. In: International Conference on Fibre Optics and Photonics, 2012 OSA Technical Digest (online) Optical Society of America TPo.19.

[12] Gupta B. D., Sharma A. K. (2005) Sensitivity evaluation of a multi-layered surface plasmon resonance-based fiber optic sensor: a theoretical study. Sens. Actuators B107(1) 40-46. 
[13] Szunerits S., Maalouli N.,Wijaya E., Vilcot J. P., Boukherroub R. (2013) Recent advances in the development of graphene-based surface plasmon resonance (SPR) interfaces. Anal. Bioanal. Chem. 405(5) 1435-1443.

[14] Zynio S. A., Samoylov A. V., Surovtseva E. R., Mirsky V. M., Shirshov Y. M. (2002) Bimetallic layers increase sensitivity of affinity sensors based on surface plasmon resonance. Sensors 2(2), 62-70.

[15] Szunerits S., Caste L. X., Boukherroub R. (2008) Surface plasmon resonance investigation of silver and gold films coated with thin indium tin oxide layers: Influence on stability and sensitivity. J. Phys. Chem. C112(40) 15813-15817.

[16] Manesse M., Sanjines R., Stambouli V., Boukherroub R., Szunerits S. (2008) Preparation and characterization of antimony-doped $\mathrm{SnO} 2$ thin films on gold and silver substrates for electrochemical and surface Plasmon resonance studies. Electrochem. Commun. 10 1041-1043.

[17] Manesse M., Sanjines R., Stambouli V., Jorel C., Pelissier B., Pisarek M., Boukherroub R., Szunerits S. (2009) Preparation and characterization of silver substrates coated with antimony-doped $\mathrm{SNO}_{2}$ thin films for surface plasmon resonance studies. Langmuir 25 8036-8041.

[18] Lockett M. R., Weibel S. C., Philips M. F., Shortreed M. R., Sun B., Corn R. M., Hamers R. J., Cerrina F., Smith L. M. (2008) Carbon-on-metal films for surface plasmon resonance detection of DNA arrays. J. Am. Chem. Soc. 130(27) 8611-8613.

[19] Touahir L., Niedziółka-Jönsson J., Galopin E., Boukherroub R., Gouget-Laemme A. C., Solomon I., Petukhov M., Chazalviel J. N., Ozanam F., Szunerits S. (2010) Surface plasmon resonance on gold and silver films coated with thin layers of amorphous silicon-carbon alloys. Langmuir 26(8) 6058-6065.

[20] Touahir L., Jenkins A. T. A., Boukherroub R., Gouget-Laemmel A. C., Chazalviel J-N., Peretti J., Ozanam F., Szunerits (2010) Surface plasmon-enhanced fluorescence spectroscopy on silver based SPR substrates. S. $J$. Phys. Chem. C114 (51) 22582-22589.

[21] Dash J. N., Jha R. (2015) On the performance of graphene-based D-shaped photonic crystal fibre biosensor using surface plasmon resonance. Plasmonics 10 1123-1131.

[22] Choi S. H., Kim Y. L., Byun K. M. (2011) Graphene-on-silver substrates for sensitive surface plasmon resonance imaging biosensors. Opt. Express 19(2) 458-466.

[23] Maharana P. K., Srivastava T., Jha R. (2013) Ultrasensitive plasmonic imaging sensor based on graphene and silicon. IEEE Photonic. Techn. Lett. 25(2) 122-125.

[24] Blake P., Brimicombe P. D., Nair R. R., Booth T. J., Jiang D., Schedin F., Ponomarenko L. A., Morozov S. V, Gleeson H. F., Hill E. W., Geim A. K., Novoselov K. S. (2008) Graphene-based liquid crystal device. Nano Lett. 8(6) 1704-1708.

[25] Castro Neto A. H., Guinea F., Peres N. M. R., Novoselov K. S., Geim A. K. (2009) The electronic properties of graphene. Rev. Mod. Phys. 81(1) 109-162.

[26] Wu L., Chu H. S., Koh W. S., Li E. P. (2010) Highly sensitive graphene biosensors based on surface plasmon resonance. Opt. Express 18(4) 14395-14400.

[27] Maharana P. K., Jha R. (2013) Enhancing performance of SPR sensor through electric field intensity enhancement using graphene. Workshop on Recent Advances in Photonics (WRAP), 17-18 Dec. 2013.

[28] Cai H.,Cui D., Zhang L. (2012) Surface plasmon resonance characteristic study of graphene-on-gold structure. In: IEEE International Conference on Nanotechnology pp. 1-3.

[29] Djeffal F., Tamersit K., Meguellati M. (2014) Analytical analysis of a new graphene-based sensor for highperformance biomolecule sensing applications. In: Proceedings of the World Congress on Engineering, July 2-4, 2014, London, U.K.

[30] Sivanandam S. N., Deepa S. N. (2008) Introduction to Genetic Algorithms. In: Computational Intelligence and Complexity.

[31] Zerari N., Mouss H. (2009) Développement d'un outil d'optimisation basé sur les algorithmes génétiques. In: 5th International Conference: Sciences of Electronic, Technologies of Information and Telecommunications, Tunisia, 22-26 March. 2009.

[32] Guo-ping W., Tadao S., Satoshi K. (2002) Sensitivity dependence of surface plasmon resonance based sensors on prism refractive index. Wuhan University Journal of Natural Science 7(1) 47-50.

[33] Yuan Y., Dai Y. (2014) A revised LRSPR sensor with sharp reflection spectrum. Sensors 14(9) 16664-16671.

[34] Benaziez N., Ounissi A., Benaziez S. (2016) Enhancement of solar cells parameters by periodic nanocylinders. Journal of Semiconductors 37(6) 064004-8.

[35] Guo-ping W., Tadao S., Satoshi K. (2002) Sensitivity dependence of surface plasmon resonance based sensors on prism refractive index. Wuhan University Journal Science 7(1) 047-050.

[36] Ferhati H., Djeffal F. (2017) Planar junctionless phototransistor: A potential high performance and low-cost device for optical communications. Optics \& Laser Technology 97 29-35.

[37] Gouicem A. M. T., Benmahammed K., Drai R., Yahi M., Taleb-Ahmed A. (2012) Multi-objective GA optimization of fuzzy penalty for image reconstruction from projections in X-ray tomography. Dig. Signal Process $22(3)$ 486-496. 
[38] Nithyarani N., Girirajkumar S. M., Anantharaman N. (2013) Modeling and control of temperature process using genetic algorithm. IJAREEIE 2(11) 5355-5364.

[39] Malhotra R., Singh N., Singh Y. (2011) Genetic algorithms: concepts, design for optimization of process controllers. Computer and Information Science 4(2) 39-54.

[40] Gen M., Cheng R., Lin L. (2008) Network models and optimization, multiobjective genetic algorithm approach. Library of Congress Control Number. 